Acta Crystallographica Section E

Structure Reports

Online

ISSN 1600-5368

\section{6-Bromo-4-oxo-4H-chromene-3-carb- aldehyde}

\section{Yoshinobu Ishikawa}

School of Pharmaceutical Sciences, University of Shizuoka, 52-1 Yada, Suruga-ku, Shizuoka 422-8526, Japan

Correspondence e-mail: ishi206@u-shizuoka-ken.ac.jp

Received 9 April 2014; accepted 9 April 2014

Key indicators: single-crystal X-ray study; $T=100 \mathrm{~K}$; mean $\sigma(\mathrm{C}-\mathrm{C})=0.004 \AA$; $R$ factor $=0.030 ; w R$ factor $=0.077 ;$ data-to-parameter ratio $=15.2$.

In the title compound, $\mathrm{C}_{10} \mathrm{H}_{5} \mathrm{BrO}_{3}$, a brominated 3-formylchromone derivative, the non- $\mathrm{H}$ atoms are essentially coplanar (r.m.s. deviation $=0.0420 \AA$ ), with the largest deviation from its mean plane [0.109 (2) ^] being found for the ring-bound carbonyl $\mathrm{O}$ atom. In the crystal, molecules are linked through halogen bonds $[\mathrm{Br} \cdots \mathrm{O}=3.191$ (2) $\AA$, $\mathrm{C}-$ $\mathrm{Br} \cdots \mathrm{O}=167.32(10)^{\circ}$ and $\left.\mathrm{C}=\mathrm{O} \cdots \mathrm{Br}=168.4(2)^{\circ}\right]$ along [101]. Molecules are assembled into layers parallel to (101) via $\pi-\pi$ stacking interactions along the $b$ axis [shortest centroidcentroid distance between the pyran and benzene rings = 3.495 (2) ̊].

\section{Related literature}

For related structures, see: Ishikawa \& Motohashi (2013); Ishikawa $(2014 a, b)$. For halogen bonding, see: Auffinger et al. (2004); Metrangolo et al. (2005); Wilcken et al. (2013); Sirimulla et al. (2013).<smiles>O=Cc1coc2ccc(Br)cc2c1=O</smiles>

\section{Experimental}

Crystal data

$\mathrm{C}_{10} \mathrm{H}_{5} \mathrm{BrO}_{3} \quad M_{r}=253.05$
Triclinic, $P \overline{1}$

$a=6.5743(18) \AA$

$b=6.967$ (3) $\AA$

$c=10.350(4) \AA$

$\alpha=71.02(3)^{\circ}$

$\beta=85.53(3)^{\circ}$

$\gamma=70.67(3)^{\circ}$

\section{Data collection}

Rigaku AFC-7R diffractometer

Absorption correction: $\psi$ scan (North et al., 1968)

$T_{\min }=0.135, T_{\max }=0.159$

2389 measured reflections

1944 independent reflections

\section{Refinement}

$R\left[F^{2}>2 \sigma\left(F^{2}\right)\right]=0.030$

$w R\left(F^{2}\right)=0.077$

$S=1.16$

1944 reflections

\author{
$V=422.8(3) \AA^{3}$ \\ $Z=2$ \\ Mo $K \alpha$ radiation \\ $\mu=4.85 \mathrm{~mm}^{-1}$ \\ $T=100 \mathrm{~K}$ \\ $0.42 \times 0.40 \times 0.38 \mathrm{~mm}$
}

Data collection: WinAFC Diffractometer Control Software (Rigaku, 1999); cell refinement: WinAFC Diffractometer Control Software; data reduction: WinAFC Diffractometer Control Software; program(s) used to solve structure: SIR92 (Altomare, et al., 1994); program(s) used to refine structure: SHELXL97 (Sheldrick, 2008); molecular graphics: CrystalStructure (Rigaku, 2010); software used to prepare material for publication: CrystalStructure.

The author acknowledges the University of Shizuoka for instrumental support.

Supporting information for this paper is available from the IUCr electronic archives (Reference: TK5306).

\title{
References
}

Altomare, A., Cascarano, G., Giacovazzo, C., Guagliardi, A., Burla, M. C., Polidori, G. \& Camalli, M. (1994). J. Appl. Cryst. 27, 435.

Auffinger, P., Hays, F. A., Westhof, E. \& Ho, P. S. (2004). Proc. Natl Acad. Sci. USA, 101, 16789-16794.

Ishikawa, Y. (2014a). Acta Cryst. E70, o439.

Ishikawa, Y. (2014b). Acta Cryst. E70, o514.

Ishikawa, Y. \& Motohashi, Y. (2013). Acta Cryst. E69, o1416.

Metrangolo, P., Neukirch, H., Pilati, T. \& Resnati, G. (2005). Acc. Chem. Res. 38, 386-395.

North, A. C. T., Phillips, D. C. \& Mathews, F. S. (1968). Acta Cryst. A24, 351359.

Rigaku (1999). WinAFC Diffractometer Control Software. Rigaku Corporation, Tokyo, Japan.

Rigaku (2010). CrystalStructure. Rigaku Corporation, Tokyo, Japan.

Sheldrick, G. M. (2008). Acta Cryst. A64, 112-122.

Sirimulla, S., Bailey, J. B., Vegesna, R. \& Narayan, M. (2013). J. Chem. Inf. Model. 53, 2781-2791.

Wilcken, R., Zimmermann, M. O., Lange, A., Joerger, A. C. \& Boeckler, F. M. (2013). J. Med. Chem. 56, 1363-1388. 


\section{supporting information}

Acta Cryst. (2014). E70, o555 [doi:10.1107/S160053681400796X]

\section{6-Bromo-4-oxo-4H-chromene-3-carbaldehyde}

\section{Yoshinobu Ishikawa}

\section{S1. Structural commentary}

Halogen bonds have been found to occur in organic, inorganic, and biological systems, and have recently attracted much attention in medicinal chemistry, chemical biology and supramolecular chemistry (Auffinger et al., 2004, Metrangolo et al., 2005, Wilcken et al., 2013, Sirimulla et al., 2013). We have recently reported the crystal structures of dihalogenated 3-formylchromone derivatives 6,8-dichloro-4-oxo-4H-chromene-3-carbaldehyde (Ishikawa \& Motohashi, 2013, Fig.3 (top left)) and 6,8-dibromo-4-oxo-4H-chromene-3-carbaldehyde (Ishikawa, 2014a, Fig.3 (top right)). It was found that halogen bonds between the formyl oxygen atom and the halogen atoms at 8-position are formed in those crystals in a similar fashion. On the other hand, halogen bond is not observed between any oxygen atom and the chlorine atom at 6position in the crystal structure of 6-chloro-4-oxo-4H-chromene-3-carbaldehyde (Ishikawa, 2014b, Fig.3 (bottom left)). As part of our interest in this type of chemical bonding, we herein report the crystal structure of a monobrominated 3formylchromone derivative 6-bromo-4-oxo- $4 H$-chromene-3-carbaldehyde. The objective of this study is to reveal whether halogen bond $(s)$ can be formed in the crystal structure of the title compound with the bromine atom at 6-position and without a halogen atom at 8-position.

The mean deviation of the least-square planes for the non-hydrogen atoms is $0.0420 \AA$, and the largest deviation is 0.109 (2) $\AA$ for O2. These mean that these atoms are essentially coplanar (Fig.1).

In the crystal, the molecules are stacked with the inversion-symmetry equivalent ${ }^{\mathrm{i}}$ along the $b$-axis direction [shortest centroid-centroid distance between the pyran and benzene rings of the $4 H$-chromene units $=3.495$ (2) $\AA$, i: $-x+1,-y+1$, $-z+1]$, as shown in Fig. 1. The $\mathrm{Cg}-\mathrm{Cg}$ distance of the title compound is almost equal to that of 6-chloro-4-oxo- $4 \mathrm{H}-$ chromene-3-carbaldehyde (3.4959 (15) ^̊, Ishikawa, 2014b).

Halogen bond was observed between the bromine atom at 6-position and the formyl oxygen atom of the translationsymmetry equivalent ${ }^{\mathrm{ti}}\left[\mathrm{Br} 1 \cdots \mathrm{O} 3^{\mathrm{ii}}=3.191\right.$ (2) $\AA$, ii: $\left.x-1, y, z+1\right]$ along [101], as shown in Fig.2. The angles of $\mathrm{C}-\mathrm{Br} \cdots \mathrm{O}$ and $\mathrm{Br} \cdots \mathrm{O}=\mathrm{C}$ are $167.32(10)$ and $168.4(2)^{\circ}$, respectively. Thus, it is found that halogen bonds are formed for the bromine atoms not only at 8-position but also at 6-position, as shown in the top right and bottom right of Fig.3.

The space group and crystal packing mode of the title compound are the same with those of 6-chloro-4-oxo- $4 H$ chromene-3-carbaldehyde. On the other hand, halogen bond is observed in the former and not in the latter, as shown in the bottom of Fig.3. These should be accounted for by the larger size of the $\sigma$ hole of the bromine atom at 6-position (Wilcken et al., 2013). These results might be applicable for rational drug design.

\section{S2. Synthesis and crystallization}

Single crystals suitable for X-ray diffraction were obtained by slow evaporation of an $N, N$-dimethylformamide solution of the commercially available title compound at room temperature. 


\section{S3. Refinement}

Crystal data, data collection and structure refinement details are summarized in Table 1 . The $\mathrm{C}\left(s p^{2}\right)$-bound hydrogen atoms were placed in geometrical positions $\left[\mathrm{C}-\mathrm{H} 0.95 \AA, U_{\mathrm{iso}}(\mathrm{H})=1.2 U_{\mathrm{eq}}(\mathrm{C})\right]$, and refined using a riding model.

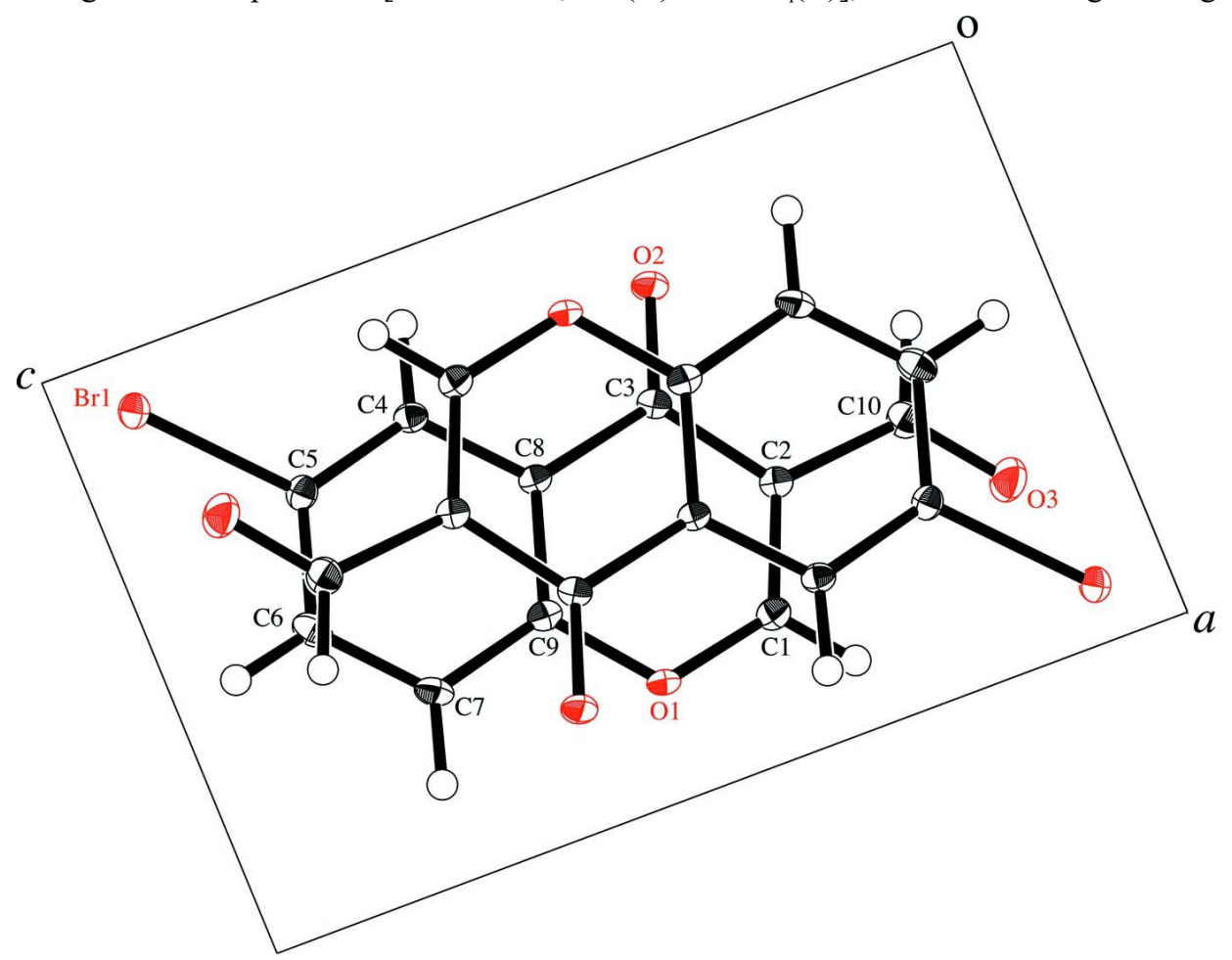

\section{Figure 1}

A packing view of the title compound with displacement ellipsoids drawn at the $50 \%$ probability level. Hydrogen atoms are shown as small spheres of arbitrary radius.
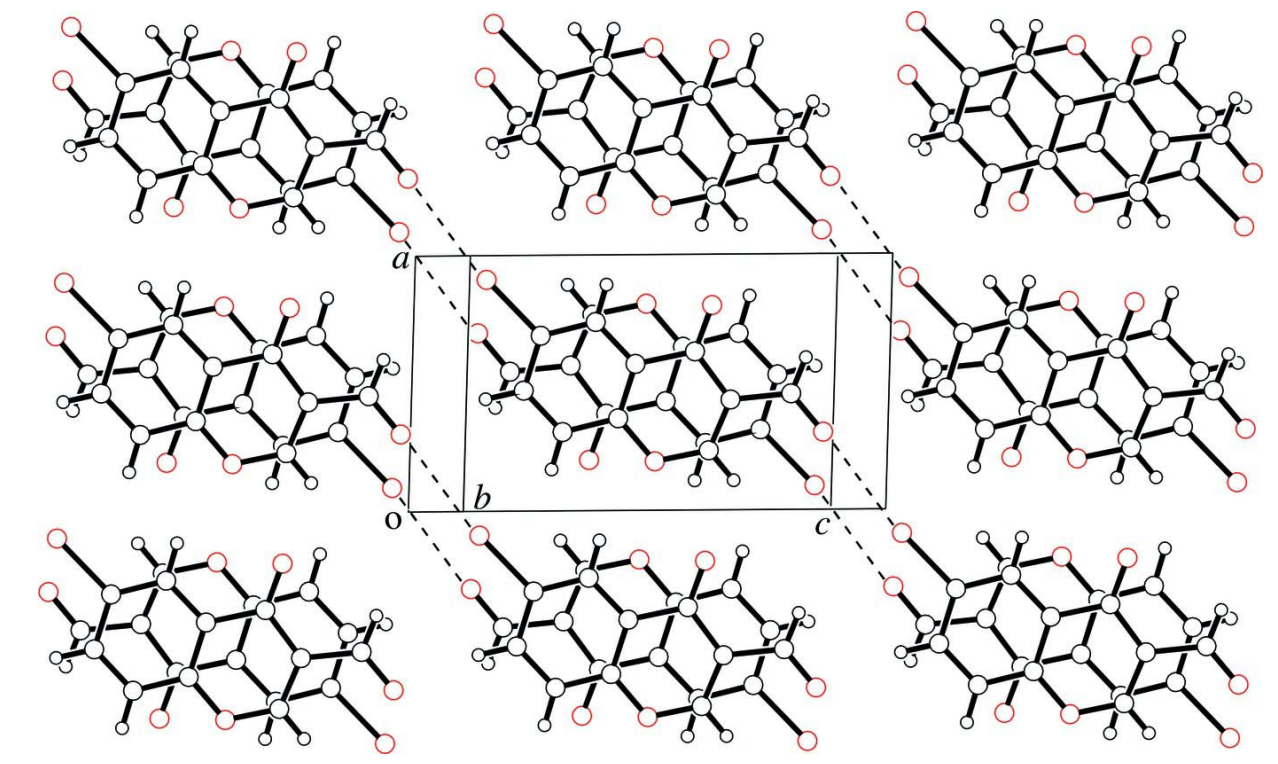


\section{Figure 2}

A packing view of the title compound. The intermolecular halogen bonds are represented as dashed lines for $\mathrm{Br} \cdots \mathrm{O}$.
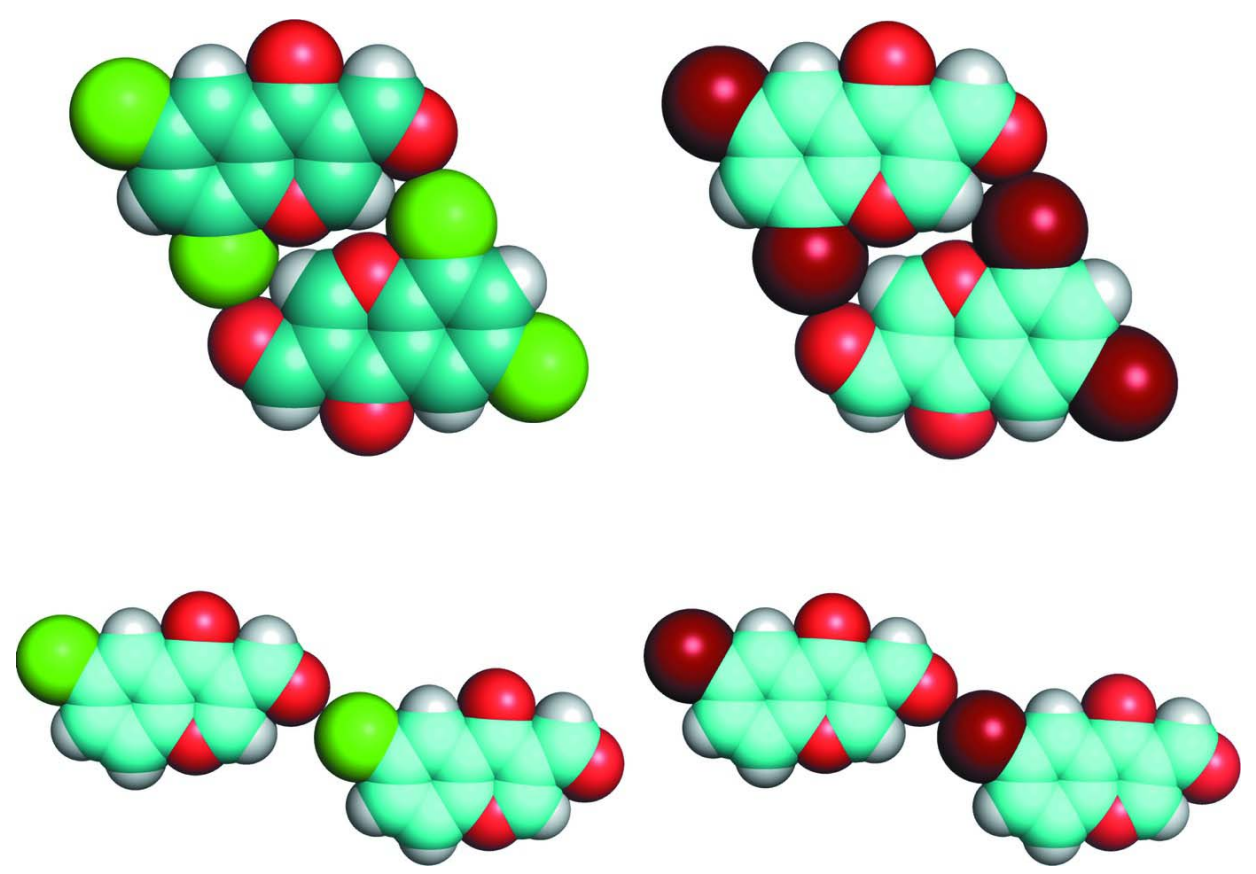

\section{Figure 3}

Sphere models of the crystal structures of 6,8-dichloro-4-oxo-4H-chromene-3-carbaldehyde (top left), 6,8-dibromo-4oxo- $4 H$-chromene-3-carbaldehyde (top right), 6-chloro-4-oxo-4H-chromene-3-carbaldehyde (bottom left), and the title compound (bottom right).

\section{6-Bromo-4-oxo-4H-chromene-3-carbaldehyde}

Crystal data

$\mathrm{C}_{10} \mathrm{H}_{5} \mathrm{BrO}_{3}$

$M_{r}=253.05$

Triclinic, $P \overline{1}$

Hall symbol: -P 1

$a=6.5743$ (18) $\AA$

$b=6.967(3) \AA$

$c=10.350(4) \AA$

$\alpha=71.02(3)^{\circ}$

$\beta=85.53(3)^{\circ}$

$\gamma=70.67(3)^{\circ}$

$V=422.8(3) \AA^{3}$

Data collection

Rigaku AFC-7R

diffractometer

$\omega-2 \theta$ scans

Absorption correction: $\psi$ scan

(North et al., 1968)

$T_{\min }=0.135, T_{\max }=0.159$

2389 measured reflections

1944 independent reflections
$Z=2$

$F(000)=248.00$

$D_{\mathrm{x}}=1.988 \mathrm{Mg} \mathrm{m}^{-3}$

Mo $K \alpha$ radiation, $\lambda=0.71069 \AA$

Cell parameters from 25 reflections

$\theta=15.2-17.4^{\circ}$

$\mu=4.85 \mathrm{~mm}^{-1}$

$T=100 \mathrm{~K}$

Block, colorless

$0.42 \times 0.40 \times 0.38 \mathrm{~mm}$

1880 reflections with $F^{2}>2 \sigma\left(F^{2}\right)$

$R_{\text {int }}=0.024$

$\theta_{\max }=27.5^{\circ}$

$h=-8 \rightarrow 8$

$k=-5 \rightarrow 9$

$l=-12 \rightarrow 13$

3 standard reflections every 150 reflections intensity decay: $2.0 \%$ 


\section{Refinement}

Refinement on $F^{2}$

$R\left[F^{2}>2 \sigma\left(F^{2}\right)\right]=0.030$

$w R\left(F^{2}\right)=0.077$

$S=1.16$

1944 reflections

128 parameters

0 restraints

Primary atom site location: structure-invariant direct methods

Secondary atom site location: difference Fourier map
Hydrogen site location: inferred from neighbouring sites

$\mathrm{H}$-atom parameters constrained

$w=1 /\left[\sigma^{2}\left(F_{\mathrm{o}}^{2}\right)+(0.0545 P)^{2}+0.1959 P\right]$

where $P=\left(F_{\mathrm{o}}^{2}+2 F_{\mathrm{c}}{ }^{2}\right) / 3$

$(\Delta / \sigma)_{\max }=0.001$

$\Delta \rho_{\max }=1.05 \mathrm{e} \AA^{-3}$

$\Delta \rho_{\min }=-0.74{\mathrm{e} \AA^{-3}}^{-3}$

Extinction correction: SHELXL97 (Sheldrick, 2008)

Extinction coefficient: $0.151(9)$

Special details

Refinement. Refinement was performed using all reflections. The weighted $R$-factor $(w R)$ and goodness of fit $(S)$ are based on $F^{2}$. $R$-factor (gt) are based on $F$. The threshold expression of $F^{2}>2.0 \sigma\left(F^{2}\right)$ is used only for calculating $R$-factor $(\mathrm{gt})$.

Fractional atomic coordinates and isotropic or equivalent isotropic displacement parameters $\left(\AA^{2}\right)$

\begin{tabular}{lllll}
\hline & $x$ & $y$ & $z$ & $U_{\text {iso }} * U_{\text {eq }}$ \\
\hline Br1 & $0.09454(3)$ & $0.28528(3)$ & $0.922893(19)$ & $0.01450(13)$ \\
O1 & $0.8085(3)$ & $0.1862(3)$ & $0.52565(16)$ & $0.0122(3)$ \\
O2 & $0.1989(3)$ & $0.3151(3)$ & $0.38347(17)$ & $0.0157(4)$ \\
O3 & $0.6969(3)$ & $0.2664(3)$ & $0.11860(18)$ & $0.0203(4)$ \\
C1 & $0.7668(4)$ & $0.2190(4)$ & $0.3943(3)$ & $0.0120(4)$ \\
C2 & $0.5687(4)$ & $0.2640(3)$ & $0.3402(2)$ & $0.0101(4)$ \\
C3 & $0.3797(4)$ & $0.2830(3)$ & $0.4253(2)$ & $0.0100(4)$ \\
C4 & $0.2621(4)$ & $0.2805(3)$ & $0.6624(2)$ & $0.0106(4)$ \\
C5 & $0.3145(4)$ & $0.2506(4)$ & $0.7956(3)$ & $0.0112(4)$ \\
C6 & $0.5287(4)$ & $0.1952(4)$ & $0.8394(3)$ & $0.0136(4)$ \\
C7 & $0.6918(4)$ & $0.1709(4)$ & $0.7478(3)$ & $0.0132(4)$ \\
C8 & $0.4269(3)$ & $0.2578(4)$ & $0.5684(2)$ & $0.0092(4)$ \\
C9 & $0.6393(4)$ & $0.2048(3)$ & $0.6128(3)$ & $0.0107(4)$ \\
C10 & $0.5462(4)$ & $0.2943(4)$ & $0.1932(3)$ & $0.0145(4)$ \\
H1 & 0.8846 & 0.2100 & 0.3347 & $0.0143^{*}$ \\
H2 & 0.1162 & 0.3159 & 0.6347 & $0.0127^{*}$ \\
H3 & 0.5614 & 0.1744 & 0.9318 & $0.0163^{*}$ \\
H4 & 0.8378 & 0.1315 & 0.7765 & $0.0158^{*}$ \\
H5 & 0.4050 & 0.3382 & 0.1550 & $0.0174 *$ \\
\end{tabular}

Atomic displacement parameters $\left(\AA^{2}\right)$

\begin{tabular}{lllllll}
\hline & $U^{11}$ & $U^{22}$ & $U^{33}$ & $U^{12}$ & $U^{13}$ & $U^{23}$ \\
\hline Br1 & $0.01323(17)$ & $0.01865(17)$ & $0.01105(16)$ & $-0.00255(10)$ & $0.00148(9)$ & $-0.00690(10)$ \\
O1 & $0.0070(7)$ & $0.0168(8)$ & $0.0124(8)$ & $-0.0032(6)$ & $-0.0008(6)$ & $-0.0044(6)$ \\
O2 & $0.0099(7)$ & $0.0254(9)$ & $0.0140(8)$ & $-0.0066(6)$ & $-0.0013(6)$ & $-0.0079(7)$ \\
O3 & $0.0184(8)$ & $0.0302(10)$ & $0.0172(9)$ & $-0.0093(7)$ & $0.0048(7)$ & $-0.0133(8)$ \\
C1 & $0.0107(9)$ & $0.0122(9)$ & $0.0133(10)$ & $-0.0034(8)$ & $0.0011(8)$ & $-0.0050(8)$ \\
C2 & $0.0104(9)$ & $0.0089(9)$ & $0.0120(10)$ & $-0.0032(7)$ & $-0.0000(8)$ & $-0.0045(7)$
\end{tabular}


supporting information

\begin{tabular}{lllllll} 
C3 & $0.0103(9)$ & $0.0085(9)$ & $0.0118(10)$ & $-0.0032(7)$ & $-0.0012(8)$ & $-0.0037(7)$ \\
C4 & $0.0095(9)$ & $0.0104(9)$ & $0.0126(10)$ & $-0.0032(7)$ & $-0.0001(8)$ & $-0.0047(8)$ \\
C5 & $0.0122(9)$ & $0.0110(9)$ & $0.0116(10)$ & $-0.0040(7)$ & $0.0019(8)$ & $-0.0055(7)$ \\
C6 & $0.0154(10)$ & $0.0122(10)$ & $0.0139(10)$ & $-0.0045(8)$ & $-0.0037(8)$ & $-0.0043(8)$ \\
C7 & $0.0107(9)$ & $0.0144(10)$ & $0.0143(11)$ & $-0.0041(8)$ & $-0.0038(8)$ & $-0.0035(8)$ \\
C8 & $0.0087(9)$ & $0.0085(8)$ & $0.0117(10)$ & $-0.0035(7)$ & $0.0001(8)$ & $-0.0043(7)$ \\
C9 & $0.0095(9)$ & $0.0088(9)$ & $0.0135(10)$ & $-0.0032(7)$ & $0.0004(8)$ & $-0.0032(7)$ \\
C10 & $0.0153(10)$ & $0.0174(10)$ & $0.0139(10)$ & $-0.0069(8)$ & $0.0005(8)$ & $-0.0075(8)$ \\
\hline
\end{tabular}

Geometric parameters $\left(\AA,{ }^{\circ}\right)$

\begin{tabular}{|c|c|c|c|}
\hline $\mathrm{Br} 1-\mathrm{C} 5$ & $1.888(3)$ & $\mathrm{C} 4-\mathrm{C} 8$ & $1.403(3)$ \\
\hline $\mathrm{O} 1-\mathrm{C} 1$ & $1.338(3)$ & $\mathrm{C} 5-\mathrm{C} 6$ & $1.401(4)$ \\
\hline $\mathrm{O} 1-\mathrm{C} 9$ & $1.375(3)$ & $\mathrm{C} 6-\mathrm{C} 7$ & $1.380(4)$ \\
\hline $\mathrm{O} 2-\mathrm{C} 3$ & $1.221(3)$ & $\mathrm{C} 7-\mathrm{C} 9$ & $1.391(4)$ \\
\hline $\mathrm{O} 3-\mathrm{C} 10$ & $1.211(3)$ & $\mathrm{C} 8-\mathrm{C} 9$ & $1.395(3)$ \\
\hline $\mathrm{C} 1-\mathrm{C} 2$ & $1.356(4)$ & $\mathrm{C} 1-\mathrm{H} 1$ & 0.950 \\
\hline $\mathrm{C} 2-\mathrm{C} 3$ & $1.461(3)$ & $\mathrm{C} 4-\mathrm{H} 2$ & 0.950 \\
\hline $\mathrm{C} 2-\mathrm{C} 10$ & $1.478(4)$ & $\mathrm{C} 6-\mathrm{H} 3$ & 0.950 \\
\hline $\mathrm{C} 3-\mathrm{C} 8$ & $1.480(4)$ & $\mathrm{C} 7-\mathrm{H} 4$ & 0.950 \\
\hline $\mathrm{C} 4-\mathrm{C} 5$ & $1.379(4)$ & $\mathrm{C} 10-\mathrm{H} 5$ & 0.950 \\
\hline $\mathrm{O} 1 \cdots \mathrm{C} 3$ & $2.868(3)$ & $\mathrm{C} 9 \cdots \mathrm{H} 1$ & 3.1828 \\
\hline $\mathrm{O} 2 \cdots \mathrm{C} 1$ & $3.572(3)$ & $\mathrm{C} 9 \cdots \mathrm{H} 2$ & 3.2719 \\
\hline $\mathrm{O} 2 \cdots \mathrm{C} 4$ & $2.869(3)$ & C9 $\cdots \mathrm{H} 3$ & 3.2502 \\
\hline $\mathrm{O} 2 \cdots \mathrm{C} 10$ & $2.894(3)$ & $\mathrm{C} 10 \cdots \mathrm{H} 1$ & 2.5537 \\
\hline $\mathrm{O} 3 \cdots \mathrm{C} 1$ & $2.820(4)$ & $\mathrm{H} 1 \cdots \mathrm{H} 5$ & 3.4881 \\
\hline $\mathrm{C} 1 \cdots \mathrm{C} 7$ & $3.574(4)$ & $\mathrm{H} 3 \cdots \mathrm{H} 4$ & 2.3392 \\
\hline $\mathrm{C} 1 \cdots \mathrm{C} 8$ & $2.757(3)$ & $\mathrm{Br} 1 \cdots \mathrm{H} 3^{\mathrm{xi}}$ & 3.1998 \\
\hline $\mathrm{C} 2 \cdots \mathrm{C} 9$ & $2.768(4)$ & $\mathrm{Br} 1 \cdots \mathrm{H} 4^{\mathrm{vi}}$ & 2.9904 \\
\hline $\mathrm{C} 4 \cdots \mathrm{C} 7$ & $2.805(4)$ & $\mathrm{Br} 1 \cdots \mathrm{H} 4^{\mathrm{xi}}$ & 3.4343 \\
\hline $\mathrm{C} 5 \cdots \mathrm{C} 9$ & $2.748(4)$ & $\mathrm{Br} 1 \cdots \mathrm{H} 5^{\mathrm{x}}$ & 3.4515 \\
\hline C6 $\cdots \mathrm{C} 8$ & 2.795 (4) & $\mathrm{Br} 1 \cdots \mathrm{H} 5^{\text {vii }}$ & 3.4131 \\
\hline $\mathrm{Br} 1 \cdots \mathrm{O} 3^{\mathrm{i}}$ & $3.191(2)$ & $\mathrm{O} 1 \cdots \mathrm{H} 1^{\mathrm{ii}}$ & 2.8201 \\
\hline $\mathrm{O} 1 \cdots \mathrm{O} 1^{\mathrm{ii}}$ & 3.117 (3) & $\mathrm{O} 1 \cdots \mathrm{H} 2^{\mathrm{iii}}$ & 2.9005 \\
\hline $\mathrm{O} 1 \cdots \mathrm{O} 2^{\mathrm{iii}}$ & $3.104(3)$ & $\mathrm{O} 1 \cdots \mathrm{H} 2^{\mathrm{v}}$ & 3.5064 \\
\hline $\mathrm{O} 1 \cdots \mathrm{O} 2^{\mathrm{iv}}$ & $3.325(3)$ & $\mathrm{O} 2 \cdots \mathrm{H} 1^{\mathrm{vi}}$ & 2.5430 \\
\hline $\mathrm{O} 1 \cdots \mathrm{C} 1^{\mathrm{ii}}$ & $3.174(3)$ & $\mathrm{O} 2 \cdots \mathrm{H} 2^{\text {vii }}$ & 2.6756 \\
\hline $\mathrm{O} 1 \cdots \mathrm{C}^{\mathrm{v}}$ & $3.479(3)$ & $\mathrm{O} 3 \cdots{ }^{\prime} 3^{\text {ix }}$ & 2.5290 \\
\hline $\mathrm{O} 1 \cdots \mathrm{C}^{\mathrm{v}}$ & $3.488(3)$ & $\mathrm{O} 3 \cdots \mathrm{H}^{\mathrm{v}}$ & 3.5734 \\
\hline $\mathrm{O} 2 \cdots \mathrm{O} 1^{\mathrm{vi}}$ & $3.104(3)$ & $\mathrm{O} 3 \cdots \mathrm{H} 4^{\mathrm{ii}}$ & 3.3419 \\
\hline $\mathrm{O} 2 \cdots \mathrm{O}^{\mathrm{iv}}$ & $3.325(3)$ & $\mathrm{O} 3 \cdots \mathrm{H} 5^{\mathrm{xii}}$ & 3.1712 \\
\hline $\mathrm{O} 2 \cdots \mathrm{C}^{\mathrm{vi}}$ & $3.113(4)$ & $\mathrm{C} 1 \cdots \mathrm{H} 2^{v}$ & 3.4876 \\
\hline $\mathrm{O} 2 \cdots \mathrm{C} 4^{\mathrm{vii}}$ & $3.325(3)$ & $\mathrm{C} 3 \cdots \mathrm{H} 2^{\text {vii }}$ & 3.4612 \\
\hline $\mathrm{O} 2 \cdots \mathrm{C} 9^{\text {iv }}$ & 3.408 (4) & $\mathrm{C} 4 \cdots \mathrm{H} 1^{\mathrm{v}}$ & 3.3664 \\
\hline $\mathrm{O} 3 \cdots \mathrm{Br} 1^{\mathrm{viii}}$ & $3.191(2)$ & $\mathrm{C} 4 \cdots \mathrm{H} 4^{\mathrm{vi}}$ & 3.2949 \\
\hline $\mathrm{O} 3 \cdots \mathrm{C} 5^{\text {iv }}$ & $3.444(4)$ & $\mathrm{C} 5 \cdots \mathrm{H} 1^{\mathrm{v}}$ & 3.3731 \\
\hline $\mathrm{O} 3 \cdots \mathrm{C} 6^{\mathrm{ix}}$ & $3.408(4)$ & $\mathrm{C} 5 \cdots \mathrm{H} 3^{\mathrm{xi}}$ & 3.2745 \\
\hline
\end{tabular}




\begin{tabular}{|c|c|c|c|}
\hline $\mathrm{C} 1 \cdots \mathrm{O} 1^{\mathrm{ii}}$ & $3.174(3)$ & $\mathrm{C} 5 \cdots \mathrm{H} 4^{\mathrm{vi}}$ & 3.5319 \\
\hline $\mathrm{C} 1 \cdots \mathrm{O} 2^{\mathrm{iii}}$ & $3.113(4)$ & $\mathrm{C} 6 \cdots \mathrm{H} 3^{\mathrm{xi}}$ & 3.0684 \\
\hline $\mathrm{C} 1 \cdots 4^{\mathrm{v}}$ & $3.285(4)$ & $\mathrm{C} 6 \cdots \mathrm{H} 5^{\text {iv }}$ & 3.5775 \\
\hline $\mathrm{C} 1 \cdots \mathrm{C}^{\mathrm{v}}$ & $3.455(4)$ & $\mathrm{C} 6 \cdots \mathrm{H} 5^{v}$ & 3.4388 \\
\hline $\mathrm{C} 1 \cdots \mathrm{C} 8^{\mathrm{v}}$ & $3.581(4)$ & $\mathrm{C} 7 \cdots \mathrm{H} 1^{\mathrm{ii}}$ & 3.4259 \\
\hline $\mathrm{C} 2 \cdots \mathrm{C} 4^{\text {iv }}$ & 3.591 (4) & $\mathrm{C} 7 \cdots \mathrm{H} 2^{\mathrm{iii}}$ & 3.2813 \\
\hline $\mathrm{C} 2 \cdots \mathrm{C}^{\mathrm{v}}$ & $3.530(4)$ & $\mathrm{C} 9 \cdots \mathrm{H} 1^{\mathrm{ii}}$ & 3.4206 \\
\hline $\mathrm{C} 2 \cdots \mathrm{C}^{\mathrm{v}}$ & $3.478(4)$ & $\mathrm{C} 9 \cdots \mathrm{H} 2^{\mathrm{iii}}$ & 3.5095 \\
\hline $\mathrm{C} 2 \cdots \mathrm{C} 7^{\mathrm{v}}$ & $3.566(4)$ & $\mathrm{C} 10 \cdots \mathrm{H} 3^{\mathrm{ix}}$ & 3.0662 \\
\hline $\mathrm{C} 2 \cdots \mathrm{C} 8^{\text {iv }}$ & 3.437 (4) & $\mathrm{C} 10 \cdots \mathrm{H} 3^{v}$ & 3.3423 \\
\hline $\mathrm{C} 3{ }^{\cdots} \mathrm{C} 3^{\text {iv }}$ & $3.568(3)$ & $\mathrm{H} 1 \cdots \mathrm{O} 1^{\mathrm{ii}}$ & 2.8201 \\
\hline $\mathrm{C} 3 \cdots \mathrm{C}^{\mathrm{v}}$ & $3.525(4)$ & $\mathrm{H} 1 \cdots \mathrm{O} 2^{\mathrm{iii}}$ & 2.5430 \\
\hline $\mathrm{C} 3 \cdots \mathrm{C} 8^{\text {iv }}$ & $3.535(4)$ & $\mathrm{H} 1 \cdots \mathrm{C}^{\mathrm{v}}$ & 3.3664 \\
\hline $\mathrm{C} 3 \cdots \mathrm{C} 9^{\text {iv }}$ & $3.588(4)$ & $\mathrm{H} 1 \cdots \mathrm{C}^{\mathrm{v}}$ & 3.3731 \\
\hline $\mathrm{C} 3 \cdots \mathrm{C} 9^{v}$ & $3.422(4)$ & $\mathrm{H} 1 \cdots \mathrm{C} 7^{\mathrm{ii}}$ & 3.4259 \\
\hline $\mathrm{C} 4 \cdots \mathrm{O} 1^{\mathrm{v}}$ & $3.479(3)$ & $\mathrm{H} 1 \cdots \mathrm{C} 9^{\mathrm{ii}}$ & 3.4206 \\
\hline $\mathrm{C} 4 \cdots \mathrm{O} 2^{\text {vii }}$ & $3.325(3)$ & $\mathrm{H} 1 \cdots \mathrm{H} 2^{\text {iv }}$ & 3.5756 \\
\hline $\mathrm{C} 4 \cdots \mathrm{C} 1^{v}$ & $3.285(4)$ & $\mathrm{H} 1 \cdots \mathrm{H} 2^{\mathrm{v}}$ & 3.4176 \\
\hline $\mathrm{C} 4 \cdots \mathrm{C} 2^{\text {iv }}$ & $3.591(4)$ & $\mathrm{H} 1 \cdots \mathrm{H} 4^{\mathrm{ii}}$ & 2.9827 \\
\hline $\mathrm{C} 4 \cdots \mathrm{C} 10^{\mathrm{iv}}$ & $3.594(4)$ & $\mathrm{H} 2 \cdots \mathrm{O} 1^{\mathrm{vi}}$ & 2.9005 \\
\hline $\mathrm{C} 5 \cdots \mathrm{O} 3^{\text {iv }}$ & $3.444(4)$ & $\mathrm{H} 2 \cdots \mathrm{O} 1^{\mathrm{v}}$ & 3.5064 \\
\hline $\mathrm{C} 5 \cdots \mathrm{C} 1^{\mathrm{v}}$ & 3.455 (4) & $\mathrm{H} 2 \cdots \mathrm{O} 2^{\text {vii }}$ & 2.6756 \\
\hline $\mathrm{C} 5 \cdots \mathrm{C} 2^{\mathrm{v}}$ & $3.530(4)$ & $\mathrm{H} 2 \cdots \mathrm{C}^{\mathrm{v}}$ & 3.4876 \\
\hline $\mathrm{C} 5 \cdots \mathrm{C} 10^{\mathrm{iv}}$ & $3.563(4)$ & $\mathrm{H} 2 \cdots \mathrm{C} 3^{\mathrm{vii}}$ & 3.4612 \\
\hline $\mathrm{C} 6 \cdots \mathrm{O} 3^{\mathrm{x}}$ & $3.408(4)$ & $\mathrm{H} 2 \cdots \mathrm{C}^{\mathrm{vi}}$ & 3.2813 \\
\hline $\mathrm{C} 6{ }^{\cdots} \cdot 2^{\mathrm{v}}$ & $3.478(4)$ & $\mathrm{H} 2 \cdots \mathrm{C} 9^{\mathrm{vi}}$ & 3.5095 \\
\hline $\mathrm{C} 6{ }^{\cdots} \mathrm{C} 10^{v}$ & $3.331(4)$ & $\mathrm{H} 2 \cdots \mathrm{H} 1^{\text {iv }}$ & 3.5756 \\
\hline $\mathrm{C} 7 \cdots \mathrm{C} 2^{\mathrm{v}}$ & $3.566(4)$ & $\mathrm{H} 2 \cdots \mathrm{H} 1^{\mathrm{v}}$ & 3.4176 \\
\hline $\mathrm{C} 7 \cdots \mathrm{C} 3^{v}$ & $3.525(4)$ & $\mathrm{H} 2 \cdots \mathrm{H} 2^{\text {vii }}$ & 3.1789 \\
\hline $\mathrm{C} 8 \cdots \mathrm{O} 1^{v}$ & $3.488(3)$ & $\mathrm{H} 2 \cdots \mathrm{H} 4^{\mathrm{vi}}$ & 2.6584 \\
\hline $\mathrm{C} 8 \cdots \mathrm{C} 1^{\mathrm{v}}$ & $3.581(4)$ & $\mathrm{H} 3{ }^{\cdots} \mathrm{Br}^{\mathrm{xi}}$ & 3.1998 \\
\hline $\mathrm{C} 8 \cdots \mathrm{C} 2^{\mathrm{iv}}$ & $3.437(4)$ & $\mathrm{H} 3 \cdots \mathrm{O}^{\mathrm{x}}$ & 2.5290 \\
\hline $\mathrm{C} 8 \cdots \mathrm{C}^{\text {iv }}$ & $3.535(4)$ & $\mathrm{H} 3 \cdots \mathrm{O} 3^{v}$ & 3.5734 \\
\hline $\mathrm{C} 8 \cdots \mathrm{C}^{\mathrm{v}}$ & $3.494(4)$ & $\mathrm{H} 3 \cdots \mathrm{C}^{\mathrm{xi}}$ & 3.2745 \\
\hline $\mathrm{C} 9 \cdots \mathrm{O} 2^{\text {iv }}$ & 3.408 (4) & $\mathrm{H} 3 \cdots \mathrm{C}^{\mathrm{xi}}$ & 3.0684 \\
\hline $\mathrm{C} 9 \cdots \mathrm{C}^{3 \mathrm{iv}}$ & $3.588(4)$ & $\mathrm{H} 3 \cdots \mathrm{C} 10^{\mathrm{x}}$ & 3.0662 \\
\hline $\mathrm{C} 9 \cdots \mathrm{C}^{\mathrm{v}}$ & $3.422(4)$ & $\mathrm{H} 3 \cdots \mathrm{C} 10^{\mathrm{v}}$ & 3.3423 \\
\hline $\mathrm{C} 9 \cdots \mathrm{C} 8^{\mathrm{v}}$ & $3.494(4)$ & $\mathrm{H} 3 \cdots{ }^{\cdots} 3^{\mathrm{xi}}$ & 2.7283 \\
\hline $\mathrm{C} 10 \cdots \mathrm{C} 4^{\mathrm{iv}}$ & $3.594(4)$ & $\mathrm{H} 3 \cdots{ }^{\prime} 5^{\mathrm{x}}$ & 2.8751 \\
\hline $\mathrm{C} 10 \cdots \mathrm{C} 5^{\text {iv }}$ & $3.563(4)$ & $\mathrm{H} 3 \cdots \mathrm{H} 5^{\mathrm{v}}$ & 3.2964 \\
\hline $\mathrm{C} 10 \cdots \mathrm{C} 6^{\mathrm{v}}$ & $3.331(4)$ & $\mathrm{H} 4 \cdots \mathrm{Br}^{\mathrm{iii}}$ & 2.9904 \\
\hline $\mathrm{Br} 1 \cdots \mathrm{H} 2$ & 2.9161 & $\mathrm{H} 4 \cdots \mathrm{Br} 1^{\mathrm{xi}}$ & 3.4343 \\
\hline $\mathrm{Br} 1 \cdots \mathrm{H} 3$ & 2.9076 & $\mathrm{H} 4 \cdots \mathrm{O}^{\mathrm{ii}}$ & 3.3419 \\
\hline $\mathrm{O} 1 \cdots \mathrm{H} 4$ & 2.5120 & $\mathrm{H} 4 \cdots \mathrm{C} 4^{\mathrm{iii}}$ & 3.2949 \\
\hline $\mathrm{O} 2 \cdots \mathrm{H} 2$ & 2.6160 & $\mathrm{H} 4 \cdots \mathrm{C} 5^{\mathrm{iii}}$ & 3.5319 \\
\hline $\mathrm{O} 2 \cdots \mathrm{H} 5$ & 2.6169 & $\mathrm{H} 4 \cdots \cdot{ }^{\mathrm{ii}}$ & 2.9827 \\
\hline $\mathrm{O} 3 \cdots \mathrm{H} 1$ & 2.4933 & $\mathrm{H} 4 \cdots \mathrm{H} 2^{\mathrm{iii}}$ & 2.6584 \\
\hline $\mathrm{C} 1 \cdots \mathrm{H} 5$ & 3.2796 & $\mathrm{H} 5 \cdots \mathrm{Br}^{\mathrm{ix}}$ & 3.4515 \\
\hline
\end{tabular}




\begin{tabular}{|c|c|c|c|}
\hline $\mathrm{C} 3 \cdots \mathrm{H} 1$ & 3.2958 & $\mathrm{H} 5 \cdots \mathrm{Br}^{\mathrm{vii}}$ & 3.4131 \\
\hline $\mathrm{C} 3 \cdots \mathrm{H} 2$ & 2.6880 & $\mathrm{H} 5 \cdots \mathrm{O} 3^{\mathrm{xii}}$ & 3.1712 \\
\hline $\mathrm{C} 3 \cdots \mathrm{H} 5$ & 2.6959 & $\mathrm{H} 5 \cdots \mathrm{C}^{\mathrm{iv}}$ & 3.5775 \\
\hline $\mathrm{C} 4 \cdots \mathrm{H} 3$ & 3.2780 & $\mathrm{H} 5 \cdots \mathrm{C}^{v}$ & 3.4388 \\
\hline $\mathrm{C} 5 \cdots \mathrm{H} 4$ & 3.2671 & $\mathrm{H} 5 \cdots \mathrm{H} 3^{\text {ix }}$ & 2.8751 \\
\hline $\mathrm{C} 6 \cdots \mathrm{H} 2$ & 3.2828 & $\mathrm{H} 5 \cdots \mathrm{H} 3^{\mathrm{v}}$ & 3.2964 \\
\hline $\mathrm{C} 8 \cdots \mathrm{H} 4$ & 3.2883 & & \\
\hline $\mathrm{C} 1-\mathrm{O} 1-\mathrm{C} 9$ & $118.51(18)$ & $\mathrm{C} 4-\mathrm{C} 8-\mathrm{C} 9$ & $118.9(2)$ \\
\hline $\mathrm{O} 1-\mathrm{C} 1-\mathrm{C} 2$ & $124.7(2)$ & $\mathrm{O} 1-\mathrm{C} 9-\mathrm{C} 7$ & $116.04(19)$ \\
\hline $\mathrm{C} 1-\mathrm{C} 2-\mathrm{C} 3$ & $120.7(2)$ & $\mathrm{O} 1-\mathrm{C} 9-\mathrm{C} 8$ & $122.2(2)$ \\
\hline $\mathrm{C} 1-\mathrm{C} 2-\mathrm{C} 10$ & $119.0(2)$ & $\mathrm{C} 7-\mathrm{C} 9-\mathrm{C} 8$ & $121.7(2)$ \\
\hline $\mathrm{C} 3-\mathrm{C} 2-\mathrm{C} 10$ & $120.3(2)$ & $\mathrm{O} 3-\mathrm{C} 10-\mathrm{C} 2$ & $124.0(3)$ \\
\hline $\mathrm{O} 2-\mathrm{C} 3-\mathrm{C} 2$ & $123.6(3)$ & $\mathrm{O} 1-\mathrm{C} 1-\mathrm{H} 1$ & 117.637 \\
\hline $\mathrm{O} 2-\mathrm{C} 3-\mathrm{C} 8$ & $122.6(2)$ & $\mathrm{C} 2-\mathrm{C} 1-\mathrm{H} 1$ & 117.636 \\
\hline $\mathrm{C} 2-\mathrm{C} 3-\mathrm{C} 8$ & $113.81(19)$ & $\mathrm{C} 5-\mathrm{C} 4-\mathrm{H} 2$ & 120.442 \\
\hline $\mathrm{C} 5-\mathrm{C} 4-\mathrm{C} 8$ & $119.1(2)$ & $\mathrm{C} 8-\mathrm{C} 4-\mathrm{H} 2$ & 120.436 \\
\hline $\mathrm{Br} 1-\mathrm{C} 5-\mathrm{C} 4$ & $119.78(16)$ & $\mathrm{C} 5-\mathrm{C} 6-\mathrm{H} 3$ & 120.165 \\
\hline $\mathrm{Br} 1-\mathrm{C} 5-\mathrm{C} 6$ & $118.71(18)$ & $\mathrm{C} 7-\mathrm{C} 6-\mathrm{H} 3$ & 120.161 \\
\hline $\mathrm{C} 4-\mathrm{C} 5-\mathrm{C} 6$ & $121.5(2)$ & $\mathrm{C} 6-\mathrm{C} 7-\mathrm{H} 4$ & 120.488 \\
\hline $\mathrm{C} 5-\mathrm{C} 6-\mathrm{C} 7$ & $119.7(3)$ & $\mathrm{C} 9-\mathrm{C} 7-\mathrm{H} 4$ & 120.487 \\
\hline $\mathrm{C} 6-\mathrm{C} 7-\mathrm{C} 9$ & $119.0(2)$ & $\mathrm{O} 3-\mathrm{C} 10-\mathrm{H} 5$ & 118.015 \\
\hline $\mathrm{C} 3-\mathrm{C} 8-\mathrm{C} 4$ & $121.2(2)$ & $\mathrm{C} 2-\mathrm{C} 10-\mathrm{H} 5$ & 118.010 \\
\hline $\mathrm{C} 3-\mathrm{C} 8-\mathrm{C} 9$ & $119.87(19)$ & & \\
\hline $\mathrm{C} 1-\mathrm{O} 1-\mathrm{C} 9-\mathrm{C} 7$ & $-179.47(17)$ & $\mathrm{C} 8-\mathrm{C} 4-\mathrm{C} 5-\mathrm{Br} 1$ & $178.91(17)$ \\
\hline $\mathrm{C} 1-\mathrm{O} 1-\mathrm{C} 9-\mathrm{C} 8$ & $-0.5(3)$ & $\mathrm{C} 8-\mathrm{C} 4-\mathrm{C} 5-\mathrm{C} 6$ & $-1.0(4)$ \\
\hline $\mathrm{C} 9-\mathrm{O} 1-\mathrm{C} 1-\mathrm{C} 2$ & $-1.9(3)$ & $\mathrm{H} 2-\mathrm{C} 4-\mathrm{C} 5-\mathrm{Br} 1$ & -1.1 \\
\hline $\mathrm{C} 9-\mathrm{O} 1-\mathrm{C} 1-\mathrm{H} 1$ & 178.1 & $\mathrm{H} 2-\mathrm{C} 4-\mathrm{C} 5-\mathrm{C} 6$ & 179.0 \\
\hline $\mathrm{O} 1-\mathrm{C} 1-\mathrm{C} 2-\mathrm{C} 3$ & $0.8(4)$ & $\mathrm{H} 2-\mathrm{C} 4-\mathrm{C} 8-\mathrm{C} 3$ & -1.5 \\
\hline $\mathrm{O} 1-\mathrm{C} 1-\mathrm{C} 2-\mathrm{C} 10$ & $-179.35(18)$ & $\mathrm{H} 2-\mathrm{C} 4-\mathrm{C} 8-\mathrm{C} 9$ & -179.7 \\
\hline $\mathrm{H} 1-\mathrm{C} 1-\mathrm{C} 2-\mathrm{C} 3$ & -179.2 & $\mathrm{Br} 1-\mathrm{C} 5-\mathrm{C} 6-\mathrm{C} 7$ & $-179.45(14)$ \\
\hline $\mathrm{H} 1-\mathrm{C} 1-\mathrm{C} 2-\mathrm{C} 10$ & 0.6 & $\mathrm{Br} 1-\mathrm{C} 5-\mathrm{C} 6-\mathrm{H} 3$ & 0.6 \\
\hline $\mathrm{C} 1-\mathrm{C} 2-\mathrm{C} 3-\mathrm{O} 2$ & $-177.1(2)$ & $\mathrm{C} 4-\mathrm{C} 5-\mathrm{C} 6-\mathrm{C} 7$ & $0.4(4)$ \\
\hline $\mathrm{C} 1-\mathrm{C} 2-\mathrm{C} 3-\mathrm{C} 8$ & $2.4(3)$ & $\mathrm{C} 4-\mathrm{C} 5-\mathrm{C} 6-\mathrm{H} 3$ & -179.6 \\
\hline $\mathrm{C} 1-\mathrm{C} 2-\mathrm{C} 10-\mathrm{O} 3$ & $5.5(4)$ & $\mathrm{C} 5-\mathrm{C} 6-\mathrm{C} 7-\mathrm{C} 9$ & $0.8(4)$ \\
\hline $\mathrm{C} 1-\mathrm{C} 2-\mathrm{C} 10-\mathrm{H} 5$ & -174.5 & $\mathrm{C} 5-\mathrm{C} 6-\mathrm{C} 7-\mathrm{H} 4$ & -179.2 \\
\hline $\mathrm{C} 3-\mathrm{C} 2-\mathrm{C} 10-\mathrm{O} 3$ & $-174.6(2)$ & $\mathrm{H} 3-\mathrm{C} 6-\mathrm{C} 7-\mathrm{C} 9$ & -179.2 \\
\hline $\mathrm{C} 3-\mathrm{C} 2-\mathrm{C} 10-\mathrm{H} 5$ & 5.4 & $\mathrm{H} 3-\mathrm{C} 6-\mathrm{C} 7-\mathrm{H} 4$ & 0.8 \\
\hline $\mathrm{C} 10-\mathrm{C} 2-\mathrm{C} 3-\mathrm{O} 2$ & $3.0(4)$ & $\mathrm{C} 6-\mathrm{C} 7-\mathrm{C} 9-\mathrm{O} 1$ & $177.51(19)$ \\
\hline $\mathrm{C} 10-\mathrm{C} 2-\mathrm{C} 3-\mathrm{C} 8$ & $-177.47(18)$ & $\mathrm{C} 6-\mathrm{C} 7-\mathrm{C} 9-\mathrm{C} 8$ & $-1.5(4)$ \\
\hline $\mathrm{O} 2-\mathrm{C} 3-\mathrm{C} 8-\mathrm{C} 4$ & $-3.2(4)$ & $\mathrm{H} 4-\mathrm{C} 7-\mathrm{C} 9-\mathrm{O} 1$ & -2.5 \\
\hline $\mathrm{O} 2-\mathrm{C} 3-\mathrm{C} 8-\mathrm{C} 9$ & $174.99(19)$ & $\mathrm{H} 4-\mathrm{C} 7-\mathrm{C} 9-\mathrm{C} 8$ & 178.5 \\
\hline $\mathrm{C} 2-\mathrm{C} 3-\mathrm{C} 8-\mathrm{C} 4$ & $177.26(17)$ & $\mathrm{C} 3-\mathrm{C} 8-\mathrm{C} 9-\mathrm{O} 1$ & $3.8(4)$ \\
\hline $\mathrm{C} 2-\mathrm{C} 3-\mathrm{C} 8-\mathrm{C} 9$ & $-4.5(3)$ & $\mathrm{C} 3-\mathrm{C} 8-\mathrm{C} 9-\mathrm{C} 7$ & $-177.29(18)$ \\
\hline
\end{tabular}


supporting information

$\begin{array}{llll}\mathrm{C} 5-\mathrm{C} 4-\mathrm{C} 8-\mathrm{C} 3 & 178.50(18) & \mathrm{C} 4-\mathrm{C} 8-\mathrm{C} 9-\mathrm{O} 1 & -177.99(18) \\ \mathrm{C} 5-\mathrm{C} 4-\mathrm{C} 8-\mathrm{C} 9 & 0.3(3) & \mathrm{C} 4-\mathrm{C} 8-\mathrm{C} 9-\mathrm{C} 7 & 1.0(4)\end{array}$

Symmetry codes: (i) $x-1, y, z+1$; (ii) $-x+2,-y,-z+1$; (iii) $x+1, y, z$; (iv) $-x+1,-y,-z+1$; (v) $-x+1,-y+1,-z+1$; (vi) $x-1, y, z$; (vii) $-x,-y+1,-z+1$; (viii) $x+1, y, z-1$; (ix) $x, y, z-1$; (x) $x, y, z+1$; (xi) $-x+1,-y,-z+2$; (xii) $-x+1,-y+1,-z$. 